\title{
In-situ Electron Holography Study of Grain Boundaries in Cerium Oxide
}

\author{
Xin $\mathrm{Xu}^{1}$, Sossina Haile ${ }^{1,2}$ and Charudatta Phatak $^{3}$ \\ 1. Applied Physics, Northwestern University, Evanston, USA. \\ 2. Department of Materials Science \& Engineering, Northwestern University, Evanston, USA. \\ 3. Materials Science Division, Argonne National Laboratory, Argonne, USA.
}

Charge transport across and along grain boundaries can have profound implications on the macroscopic behavior of materials used in solid oxide fuel cells, batteries as well as other energy technologies. The grain boundary may serve as high conductivity pathway or roadblock for ionic or electronic carriers. Even pristine grain boundaries, free of impurity species or secondary phases, can display modified transport properties relative to the bulk as a result of space charge effects. This is particularly true of doped ceria, which is a leading candidate for a range of applications due to fast oxygen ion conduction in the bulk. To date, the vast majority of grain boundary studies have relied on macroscopic measurements that yield ensemble averages [1]. However, a fundamental understanding of their behavior requires access to the properties of individual grain boundaries, in terms of both chemistry and electrical profiles. Electron holography offers an excellent combination of high spatial resolution and sensitivity to measure mean inner potential as well as grain boundary potential in these materials.

The goal of our work is to perform direct measurement of the inner potential in the grain boundary region of Sm0.15Ce0.85O2- $\delta$ (SDC) using electron holography. The ceria sample $(99.9 \%$, SigmaAldrich) was prepared by pressing and sintering at $1500^{\circ} \mathrm{C}$ for 10 hours and followed by standard TEM specimen preparation techniques of polishing and Ar ion milling. We performed electron holography on the grain boundary region of as-prepared polycrystalline ceria during an in-situ heating experiment where the sample was heated to $300^{\circ} \mathrm{C}$. The off-axis electron holography was performed using Tecnai F20 TEM at the Center for Nanoscale Materials at Argonne National Laboratory. The holography experiments were performed with a biprism bias of $100 \mathrm{~V}$ which yielded a good fringe contrast $(>20 \%)$ and spatial resolution $(\sim 4 \mathrm{~nm})$ during the entire temperature range. Diffraction contrast was carefully reduced by tilting the grains away from the zone axis [2]. The grain boundary potential was calculated from the measured phase shift of the electrons by accounting for the thickness of the sample. The hologram, measured phase shift and inner potential of a grain boundary are shown in Figure 1.

Simulations based on space charge theory [3] were performed to understand the dependence of GB potential and width as a function of temperature. The charge transport measurements using AC impedance spectroscopy [4] on the same batch of ceria sample combined with results from simulations were found to agree well with the holography results. We were able to confirm that the as-measured GB barrier potential and width were indeed related to the space charge potential and space charge layer thickness. We investigated different types of grain boundaries with varying misorientation, and the results showed that grain boundaries with higher misorientation angle about [110] axis showed larger potential. We additionally performed orientation imaging and atom probe tomography to determine the causes for the larger grain boundary potential measured. We will discuss these results and the observed trends in the grain boundary potential. This study showed that electron holography can be successfully used for measuring space charge effect at the grain boundaries in ionic conductors such as ceria and will help understand the charge transport mechanism of grain boundaries in polycrystalline cerium oxide samples [5]. 


\section{References:}

[1] X. Guo, J. Maier, J. Electrochem. Soc., 148(3) (2001), E121-E126.

[2] V. Ravikumar, R. P. Rodrigues, V. P. Dravid, J. Amer. Cer. Soc. 80(5) (1997), p.1117.

[3] S. Kim, J. Fleig, J, Phys. Chem. Chem. Phys, 5(11) (2003), p. 2268.

[4] W. C. Chueh et al, Phys. Chem. Chem. Phys, 13(14) (2011), p. 6442.

[5] This work was supported by the National Science Foundation's MRSEC program (DMR-1121262)

at the Materials Research Center of Northwestern University. CP would also like to acknowledge support by Northwestern-Argonne Early Career Investigator Award for Energy Research. Use of the Center for Nanoscale Materials, an Office of Science user facility, was supported by the U.S.

Department of Energy, Office of Science, Office of Basic Energy Sciences, under Contract No. DEAC02-06CH11357.
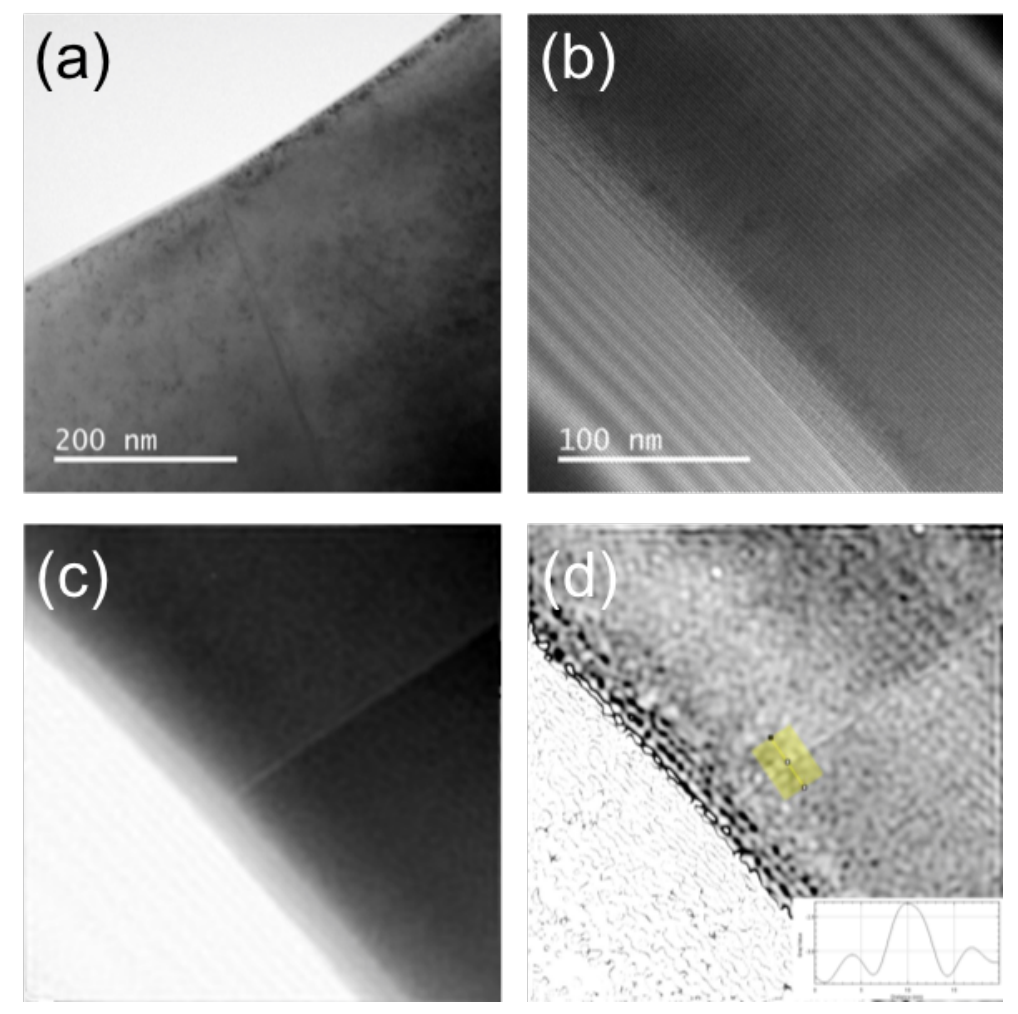

Figure 1. (a) TEM image showing the location of the grain boundary, (b) Hologram acquired from the same region, (c) the recovered phase shift across the grain boundary, and (d) the map of potential with a line plot across the grain boundary shown inset. 